\title{
Ab initio calculation of the Structural, Mechanical and Thermodynamic Properties of Beryllium Sulphide (BeS)
}

\author{
${ }^{1 * I Y O R Z O R, ~ B E ; ~}{ }^{1}$ BABALOLA, MI; ${ }^{2}$ AIGBEKAEN, EE
}

\author{
${ }^{I}$ Department of Physics, University of Benin, Benin City. Nigeria. \\ ${ }^{2}$ Department of Physics, Igbinedion University, Okada, Edo State. Nigeria. \\ *Corresponding Author: B. E. Iyorzor, Email: beniyorzor@uniben.edu
}

\begin{abstract}
An $a b$ initio plane-wave Pseudopotential calculations using the density functional theory (DFT) implementing the generalised gradient approximation (GGA) to study the structural, elastic constants, phonon dispersion curves, density of state and thermal properties of BeS. Also we calculated the shear modulus, Young's modulus, Poisson's ratio, and the Zener's anisotropic factors. The calculated properties are agreement with previous experimental and theoretical results. The quasi-harmonic approximation is applied to determine the thermal properties, and these properties are in good agreement with available literatures. The major results of the properties determined were discussed.
\end{abstract}

\section{DOI: $\quad$ https://dx.doi.org/10.4314/jasem.v22i1.8}

Copyright: Copyright (C) 2017 Iyorzor et al. This is an open access article distributed under the Creative Commons Attribution License (CCL), which permits unrestricted use, distribution, and reproduction in any medium, provided the original work is properly cited

Dates: Received 05 November 2017; received in revised form 09 December 2017; accepted 22 December 2017

Keywords: Poisson's ratio, Plane-Wave Pseudopotential, Quasi-Harmonic Approximation, Lattice Parameter,

INTRODUCTION As shown by an earlier study (Camp and Doren 1996) the alkaline earth sulphide $\mathrm{BeS}$ has unique properties that are quite different from those of the other compounds $\mathrm{BeX}(\mathrm{X}=\mathrm{O}, \mathrm{Se}, \mathrm{Te})$ in the series. Also, according to Yim et al, (1972), Zachariasen $(1926)^{\mathrm{a}-\mathrm{b}}$ and Staritzky (1956), the beryllium chalcogenides elements only BeS, BeSe and BeTe have the structure of the zinc-blende (ZB), which is a common feature to many well-known II VI and III - V semiconductors. In the works of (Luo et al (1994); (1995), Weir et al (1986) and Vassiliou and Ahrens (1981)), it was observed recently that pressure induced structural phase transition in $\mathrm{SrSe}$, calcium chalcogenides (CaS, CaSe, CaTe) and MgTe have left the beryllium chalcogenides (BeS, BeSe, BeTe) and mangnesium chalcogenides ( $\mathrm{MgS}, \mathrm{MgSe}, \mathrm{MgTe})$ the only unknown members in the entire IIA - VI series in regards to high pressure phase transition sequence. Srivastava et al (2004), in their work, did a study on the structural, electronic and dynamical properties of Be-Chalcogenide materials by using the plane wave pseudopotentials, density functional theory, and a linear response approach. The structural phase transition and ground state properties, and pressure dependence of the band gap of the series of compounds BeS, BeSe and BeTe have been calculated by employing the first principles tight binding linear muffin-tin orbital method within the local density approximation (LDA) Kalpana et al (1998). Also the results of these calculated bulk modulus the pressure volume relation and the lattice parameters, were found to be in good agreement with the recent experimental results. In recent times, the fast growing research areas includes investigation of thin layers and multilayers with different alloys containing beryllium. In the works of Paszkowicz et al (1998), they report that a partial replacement of $\mathrm{Zn}$ with $\mathrm{Be}$ improves the properties of the device because of dominant covalent bonding and high cohesive energy of BeSe. Such substitution allows to extend the domain of structural and band-gap engineering of II-VI semiconductors towards lower lattice parameters and higher energy gaps. In the past decade, the electronic and structural properties of solids from first principle calculations has become possible to compute with great accuracy. From these computations the lattice constants, crystal structures, phonon spectra, bulk and shear moduli and other static and dynamical properties can be obtained and it is also possible to predict the properties of solid, which were formally not experimentally accessible. As a function of temperature, Lee and Xavier (1995), calculated the constant volume specific heat, the entropy, the phonon contributions to internal energy and Helmholts free energy and the atomic temperature factors of $\alpha$-quartz and stishovite, two allotropic forms of $\mathrm{SiO}_{2}$ from $a b$ initio phonon band structures. To determine the phonon density of state, dispersion relations, decomposition density of states and thermal 
quantities, Laref and Laref (2012) in their work, used the density functional perturbations theory with quasiharmonic approximation QHA methods. In our present paper, we used an $a b$ initio and quasi-harmonic approximations to study the structural, mechanical and thermodynamic properties of $\mathrm{BeS}$ using the density functional theory as viewed from the xcrysden software presented in Fig. 1.

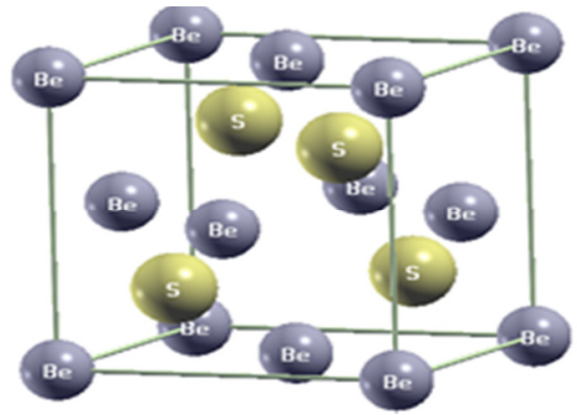

Fig. 1. Crystal structure of the BeS. The Be atoms form a facecentered-cubic (fcc) lattice.

\section{MATERIALS AND METHODS}

Total Energy Electronic Structure Calculation: The first-principles calculations were performed using the QUANTUM ESPRESSO code (QE) as implemented in Baroni et al (2009), which has a basis set of a pane wave Pseudopotential PWPP method with the generalized gradient approximation GGA as reported in the works of Perdew et al (1996). In order to achieve convergence, a plane wave basis set with kinetic energy cut off of $612 \mathrm{eV}$, charge density cut off of $2449 \mathrm{eV}$ and convergence threshold of $1.0 \times 10^{-8}$ were applied and for the brillouin zone sampling, $8 \times 8 \times 8$ Monkhorst - Pack meshes for BeS was used. During the self-consistent calculation, the data set generated from the energy lattice parameter was fitted to the Birch third order Murnaghan equation of state and the equilibrium lattice constant, bulk modulus and pressure derivate of the bulk modulus were obtained.

Elastic Properties: We obtained the elastic constants after performing energy variation due to the applications of little strain to an optimized unit cell. According to Mehl (1993) and Wang and Ye (2003), the energy of a solid under stain is given as

$\delta E=E_{t o t}-E_{0}=\frac{V}{2} \sum_{i=0}^{6} \sum_{j=0}^{6} c_{i j} e_{i} e_{j}$

where $v$ is the volume of the equilibrium lattice cell, $\delta E$ is the energy increment from the strain with vector $e=\left(e_{1}, e_{2}, e_{3}, e_{4}, e_{5}, e_{6}\right)$ and $c$ is the matrix of the elastic constants. For cubic phases there are three independent elastic constants, $c_{11}, c_{12}$ and $c_{44}$. Its primitive vectors are defined as;

$$
\left(\begin{array}{l}
a_{1} \\
a_{2} \\
a_{3}
\end{array}\right)=\left(\begin{array}{ccc}
0 & \frac{\alpha}{2} & \frac{\alpha}{2} \\
\frac{\alpha}{2} & 0 & \frac{\alpha}{2} \\
\frac{\alpha}{2} & \frac{\alpha}{2} & 0
\end{array}\right)
$$

where $a$ is the lattice constant and the primitive vectors $a_{1}, a_{2}, a_{3}$ are transformed to the new vectors under strain by;

$$
\left(\begin{array}{l}
a_{1}^{\prime} \\
a_{2}^{\prime} \\
a_{3}^{\prime}
\end{array}\right)=\left(\begin{array}{l}
a_{1} \\
a_{2} \\
a_{3}
\end{array}\right)(I+\varepsilon)
$$

Where $\varepsilon$ is the strain tensor and $I$ is the identify matrix and it is related to the strain vector $e$ by;

$$
\varepsilon=\left(\begin{array}{ccc}
e_{1} & \frac{e_{6}}{2} & \frac{e_{5}}{2} \\
\frac{e_{6}}{2} & e_{2} & \frac{e_{4}}{2} \\
\frac{e_{5}}{2} & \frac{e_{4}}{2} & e_{3}
\end{array}\right)
$$

We apply the tri-axial shear strain $e=(0,0,0, \delta, \delta, \delta)$ to the crystal in the calculation of the elastic constants of the cubic phase. The $c_{44}$ can be calculated from;

$\frac{\Delta E}{v}=\frac{3}{2} c_{44} \delta^{2}$

Also the shear modulus $G=\frac{1}{2}\left(c_{11}-c_{12}\right)$ was obtained from the volume - conserving orthorhombic strain $\varepsilon=\left(\delta, \delta,(1+\delta)^{-2}-1,0,0,0\right)$ by using;

$\frac{\Delta E}{v}=6 G \delta^{2}$

Hence $c_{11}$ and $c_{12}$ are given by;

$$
\begin{aligned}
& c_{11}=\frac{3 B+4 G}{3} \\
& c_{12}=\frac{3 B-2 G}{3}
\end{aligned}
$$

Once the three independent elastic constants for cubic phases, $c_{11}, c_{12}$ and $c_{44}$, have been calculated parameters such as the Poisson's ratio $\mathcal{V}$, Young modulus $E$ and Zener anisotropy factor $A$ can be obtained from the following expressions:

$$
\begin{aligned}
\mathrm{V} & =\frac{3 B-2 G}{2(3 B+G)} \\
E & =\frac{9 G B}{3 B+G} \\
A & =\frac{2 c_{44}}{c_{11}-c_{12}}
\end{aligned}
$$

The relationship between the mechanical and dynamic behavior of the crystals can obtained from elastic constants, also vital information about the nature of the forces operating in the solids and the stability and 
stiffness of the materials can be obtained. Hence, from the work of (Sinko and Smirnow 2002), for cubic crystals, the mechanical stability is measured by the following conditions: $c_{11}+2 c_{12}>0, c_{11}-c_{12}>0$ ， $c_{44}>0$, and $c_{11}>0,(12)$

Thermodynamic Properties: The thermodynamic properties of BeS can be calculated by applying the quasi harmonic Debye model as reported in Chang et al., (2008). In the quasi-harmonic Debye model, the non-equilibrium Gibbs function $G *(V ; P, T)$ is taken in the form of:

$G *(V ; P, T)=E(V)+P V+A_{v i b}(\Theta(V) ; T)$

where $E(V)$ is the total energy, $P V$ corresponds to the constant hydrostatic pressure condition $\Theta(V)$ is the Debye temperature. The adiabatic bulk modulus is given by;

$B_{s}=B(V)=V\left\{\frac{d^{2} E(V)}{d V^{2}}\right\}$

the isothermal bulk modulus $B_{T}$ and heat capacity $c_{v}$

$B_{T}(P, T)=V\left(\frac{\partial^{2} G *(V ; T, P)}{\partial V^{2}}\right)_{P, T}$

and $c_{v}=3 n K_{B}\left[4 D(\Theta / T)-\frac{3 \Theta / T}{e^{\Theta / T}-1}\right]$

RESULTS AND DISCUSSION

Structural Properties: The equilibrium lattice constant (a) has been determined by calculating the total energy at a range of lattice parameters around the experimental value within PWPP method with the generalized gradient approximation GGA, Perdew et al., (1996). We obtained the equilibrium lattice parameter $a$, the bulk modulus $B$ and the pressure derivative of the bulk modulus $B^{\prime}$ by applying the fitting of the third order Birch-Mumaghan equation of state on the energy lattice parameters. We summarized our results, experimental values and other theoretical values as recorded in the works of Staritzky, (1956), Heciri et al, (2007), Narayana et al (1997), Laref and Laref, (2012), Guo et al., (2013), Okoye, (2004),
Khenata et al., (2006), Benosman et al., (2001), and Gonzalez-Diaz et al., (1997) presented in Table 1(i). The optimized lattice parameter for the BeS compound in this work is consistent with the experimental values, although with slight discrepancy of about $0.0086 \%$.

Table 1. Calculated lattice constant $a(\AA)$, bulk modulus $B(G P a)$ and its pressure-derivative $B^{\prime}$ for BeS compared with other experimental and theoretical results. (i) (Structural Properties)

\begin{tabular}{llll}
\hline Material $(\mathrm{BeS})$ & $a(\AA)$ & $B(G P a)$ & $B^{\prime}$ \\
\hline Present work & 4.862 & 92.7 & 3.67 \\
Experimental" & $4.862^{\mathrm{a}}$ & --- & --- \\
& $4.870^{\mathrm{b}}$ & -- & -- \\
Other theoretical & $4.878^{\mathrm{c}}$ & $93^{\mathrm{c}}$ & $3.524^{\mathrm{c}}$ \\
calculations & $4.83^{\mathrm{d}}$ & $93^{\mathrm{d}}$ & $3.33^{\mathrm{d}}$ \\
& $4.88^{\mathrm{e}}$ & $93.1^{\mathrm{e}}$ & $3.75^{\mathrm{e}}$ \\
& $4.88^{\mathrm{f}}$ & $92.23^{\mathrm{f}}$ & $3.07^{\mathrm{f}}$ \\
& $4.80^{\mathrm{g}}$ & $100.8^{\mathrm{g}}$ & $3.63^{\mathrm{g}}$ \\
& $4.819^{\mathrm{h}}$ & $1.13(\mathrm{Mbar})^{\mathrm{h}}$ & $3.99^{\mathrm{h}}$ \\
\hline
\end{tabular}

$\mathrm{a}=$ Staritzky, (1956); b = Narayana et al, (1997), c= Heciri et al, (2007), d=Laref and Laref (2012), e=Guo et al, (2013), f=Okoye, (2004), g=Khenata et al, (2006), h = Benosman et al, (2001),

$\mathrm{i}=$ Gonzalez-Diaz et al, (1997)

Mechanical Properties: The investigation of the elastic constant requires knowledge of the shape of the energy curves as function of strain for unit cell. To determine the mechanical properties we have used the PWPP scheme following the method implemented in $\mathrm{QE}$, by finite strain technique. We obtained values for the elastic constants: $c_{11}, c_{12}, c_{44}$, the Zener anisotropy $A$, the Poisson's ratio $\mathrm{V}$, Young modulus $E$ and the shear modulus $G$ by applying the equations (7-11) as stated in section 2.2. Our calculated values are in good agreement with previous theoretical results (See Table 1 and 2). The slight deviations are partly due to the calculation methods and fitting errors. And the stability conditions as in (Sinko and Smirnow, 2002) are all satisfied, which indicate that the compound $\mathrm{BeS}$ is mechanically stable at zero pressure and obeys the cubic stability conditions.

Table 2. Elastic constants: $c_{11}, c_{12}, c_{44}$, the Zener anisotropy $A$, the Poisson's ratio $\mathrm{V}$, Young modulus $E$ and the shear modulus

\begin{tabular}{|c|c|c|c|c|c|c|c|}
\hline Material & $C_{11}\left(\begin{array}{lll}G P & a\end{array}\right)$ & $C_{12}(G P a)$ & $C_{44}\left(\begin{array}{lll}P & a\end{array}\right)$ & $A$ & $\mathrm{~V}$ & $E$ & $G$ \\
\hline \multicolumn{8}{|l|}{$\mathrm{BeS}$} \\
\hline Present work & 159.82 & 59.14 & 88.48 & 1.76 & 0.27 & 127.87 & 50.34 \\
\hline Experimental & --- & --- & --- & --- & --- & --- & --- \\
\hline Other theoretical & $146^{\mathrm{c}}$ & $67^{\mathrm{c}}$ & $103^{\mathrm{c}}$ & --- & --- & --- & --- \\
\hline calculations & $157^{\mathrm{d}}$ & $61^{\mathrm{d}}$ & $97^{\mathrm{d}}$ & --- & $0.19^{\mathrm{d}}$ & $174^{\mathrm{d}}$ & $73^{\mathrm{d}}$ \\
\hline , & $158.2^{\mathrm{e}}$ & $61.1^{\mathrm{e}}$ & $92.7^{\mathrm{e}}$ & --- & --- & --- & --- \\
\hline , & $167^{\mathrm{g}}$ & $68^{\mathrm{g}}$ & $105^{\mathrm{g}}$ & --- & --- & --- & --- \\
\hline , & $1.87(\mathrm{Mbar})^{\mathrm{h}}$ & $0.75(\mathrm{Mbar})^{\mathrm{h}}$ & $0.82(\mathrm{Mbar})^{\mathrm{h}}$ & --. & --- & --- & --- \\
\hline , & $1.84(\mathrm{Mbar})^{\mathrm{i}}$ & $0.75(\mathrm{Mbar})^{\mathrm{i}}$ & 0.99 (Mbar $^{\mathrm{i}}$ & --- & --- & --- & --- \\
\hline
\end{tabular}


Table 3. Calculated phonon frequencies $\left(\mathrm{in}^{-1}\right.$ ) at the high-symmetry points $\Gamma, X, W$, and $L$ compared with other theoretical

\begin{tabular}{lllllllllll}
\multicolumn{10}{c}{ results. } \\
\hline Material BeS & LO $(\Gamma)$ & TO $(\Gamma)$ & LO $(X)$ & TO $(X)$ & LA $(X)$ & LA $(X)$ & LO (L) & TO (L) & LA (L) & LA (L) \\
\hline Present work & 524 & 524 & 647 & 493 & 370 & 240 & 601 & 531 & 371 & 173 \\
Other theoretical & $655^{\mathrm{a}}$ & $566^{\mathrm{a}}$ & $611^{\mathrm{a}}$ & $548^{\mathrm{a}}$ & $364^{\mathrm{a}}$ & $163^{\mathrm{a}}$ & $656^{\mathrm{a}}$ & $513^{\mathrm{a}}$ & $367^{\mathrm{a}}$ & $229^{\mathrm{a}}$ \\
calculation & $647^{\mathrm{b}}$ & $562^{\mathrm{b}}$ & $652^{\mathrm{b}}$ & $507^{\mathrm{b}}$ & $364^{\mathrm{b}}$ & $237^{\mathrm{b}}$ & $607^{\mathrm{b}}$ & $543^{\mathrm{b}}$ & $362^{\mathrm{b}}$ & $161^{\mathrm{b}}$ \\
& $652^{\mathrm{c}}$ & $562^{\mathrm{c}}$ & $654^{\mathrm{c}}$ & $505^{\mathrm{c}}$ & $357^{\mathrm{c}}$ & $226^{\mathrm{c}}$ & $607^{\mathrm{c}}$ & $545^{\mathrm{c}}$ & $361^{\mathrm{c}}$ & $158^{\mathrm{c}}$ \\
& $647^{\mathrm{d}}$ & $562^{\mathrm{d}}$ & $635^{\mathrm{d}}$ & $585^{\mathrm{d}}$ & $372^{\mathrm{d}}$ & $258^{\mathrm{d}}$ & $636^{\mathrm{d}}$ & $577^{\mathrm{d}}$ & $383^{\mathrm{d}}$ & $179^{\mathrm{d}}$ \\
\hline
\end{tabular}

Table 4. Calculated values of $S, C_{v}, \Theta_{D}, \Delta E$ and $\Delta F$ at room temperature.

\begin{tabular}{lccccc}
\hline Material, BeS & $S(J / m o l . K)$ & $C_{v}(J / m o l . K)$ & $\Theta_{D}(K)$ & $\Delta E(J)$ & $\Delta F(J)$ \\
\hline Present work & 33.8 & 43.3 & 791 & 0.015 & 0.0087 \\
Other theoretical calc. & $30.7^{\mathrm{d}}$ & $36.3^{\mathrm{d}}$ & $782^{\mathrm{d}}$ & --- & --- \\
\hline
\end{tabular}

Phonon Dispersion Curves and Density of States: We present the calculated phonon dispersion curves and the density of states curves in Fig 2 and Fig 3 respectively. In Table 3 , our results presented are in good agreement when compared with available theoretical values. The longitudinal and transverse frequency modes of optical branch at $\Gamma$ point are in good agreement with the work of (Laref and Laref, 2012) but with slight deviation in the longitudinal optical. We noticed some features in the dispersion: Along the symmetry direction $\Gamma_{-L}$ the longitudinal optical $L O$ branch shows a sharp increasing amount of upward dispersion while the $T O$, a flat/horizontal dispersion it was also observed that the $L O$ and $T O$ branches did not cross. The calculated frequencies $T O$ and $L O$ at $\Gamma$ point coincide at $524 \mathrm{~cm}^{-1}$ in this work, while the work of (Laref and Laref, 2012), there is a separation between $T O$ and $L O$ and they are $566 \mathrm{~cm}^{-1}$ and $655 \mathrm{~cm}^{-1}$ respectively, also from the work of Srivastava et al, (2004) the frequencies of $T O$ and $L O$ at $\Gamma$ point are $562 \mathrm{~cm}^{-1}$ and $647 \mathrm{~cm}^{-1}$. The disparity may be due to the type of k-point used in our calculation.

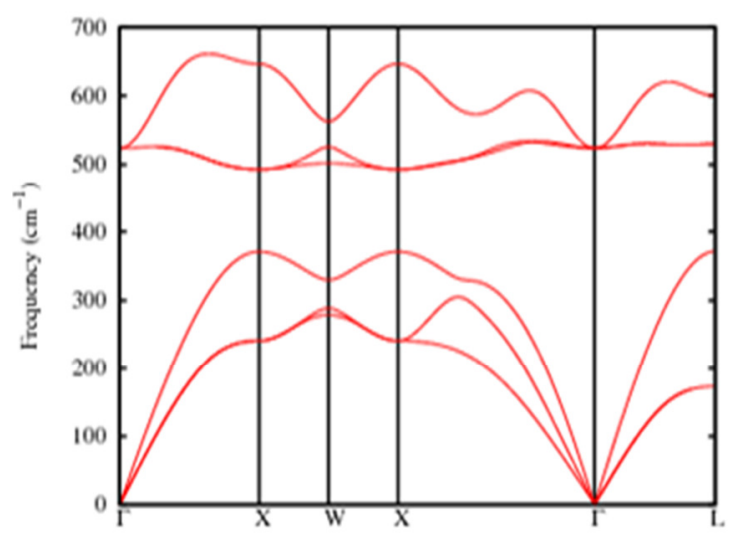

Fig. 2 Calculated phonon dispersions curve for BeS.

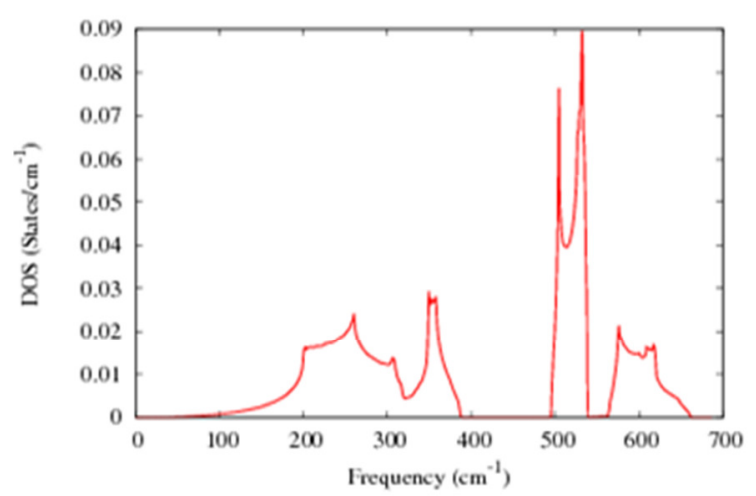

Fig. 3. Phonon density of states for BeS

Thermodynamic Properties: The thermodynamic properties of BeS have been investigated successfully. Figs $4-8$ show the results of the Debye temperature $\Theta_{D}$, vibrational entropy $S$, specific heat at constant volume $C_{v}$, Free energy $\Delta F$, and the internal energy $\Delta E$ of the compound within a temperature range of 0 to $500 \mathrm{~K}$. At high temperature $S$ and $C_{v}$ values increase sharply and eventually tends to a constant beyond $500 \mathrm{~K}$. From the graph of Free energy $\Delta F$, with increasing temperature, the Free energy values reduce quickly and approaching zero beyond $500 \mathrm{~K}$, while the internal energy increases slowly with temperature. At room temperature $(300 \mathrm{~K})$, the calculated values of the $S, C_{v}, \Theta_{D}, \Delta E$ and $\Delta F$ are presented in Table 4. Also the Debye temperature $\Theta_{D}$ at minimum and $T \rightarrow 0$ are $684 K$ and $970 K$ is presented in Table 5 .

Table 5. Debye temperature ( $\Theta_{D_{(\min )}}$ and $\Theta_{D_{(T \rightarrow 0)}}$ ) for BeS when compared with other theoretical results

\begin{tabular}{|c|c|c|}
\hline Material & $\Theta_{D_{(\min )}}(K)$ & $\Theta_{D_{(T \rightarrow 0)}}(K)$ \\
\hline \multicolumn{3}{|l|}{$\mathrm{BeS}$} \\
\hline Present work (GGA) & 684 & 970 \\
\hline Other theoretical calc. (LDA) & $608^{d}$ & $748^{d}$ \\
\hline$\left(\mathrm{LDT}^{+}\right.$ & $615^{\mathrm{k}}$ & $742^{\mathrm{k}}$ \\
\hline
\end{tabular}

+ (k) Lattice dynamic theory, Wang and Ye, (2003) 


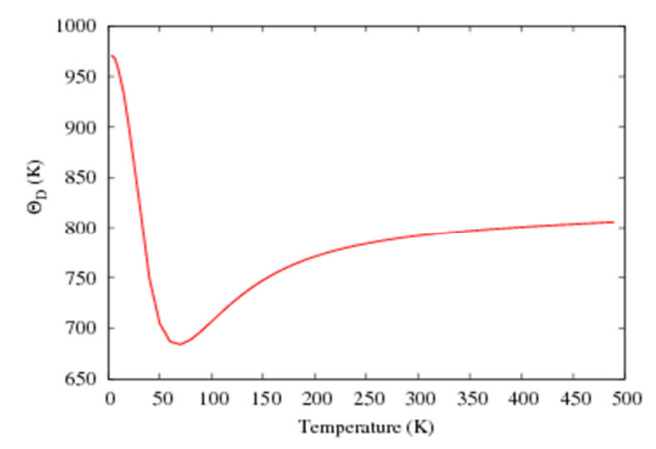

Fig. 4. The calculated Debye temperature within the range of $(0-500 \mathrm{~K})$

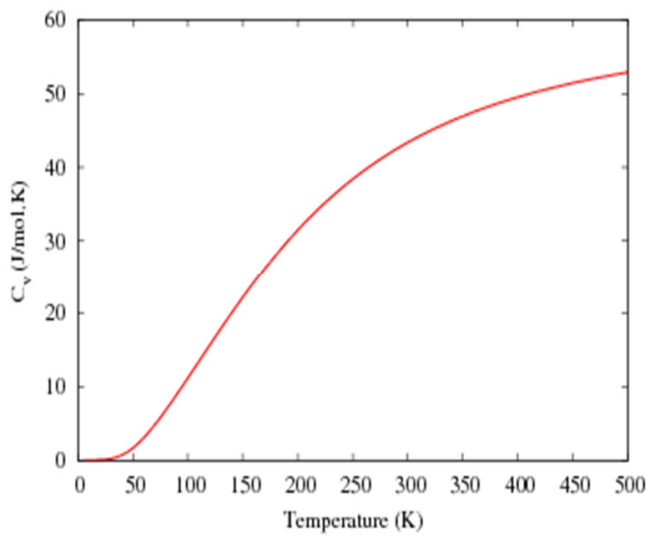

Fig. 5. Calculated specific heat at constant volume $\mathrm{Cv}(\mathrm{J} / \mathrm{mo} . \mathrm{K})$.

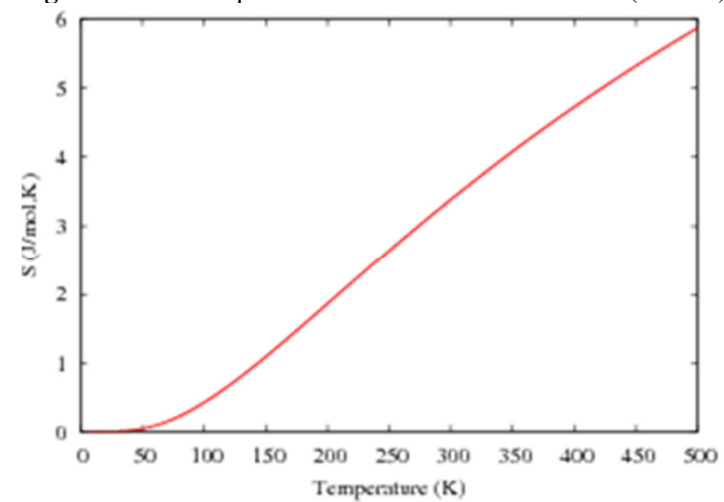

Fig. 6. The calculated vibrational entropy $S$ ( $J / m o l . K)$.

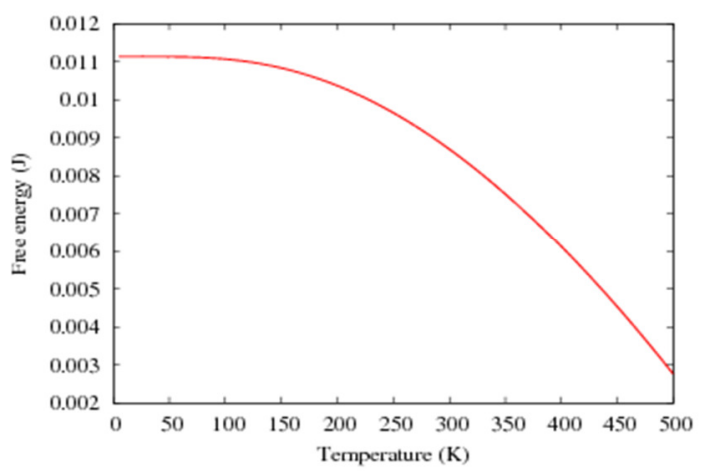

Fig. 7. The calculated Free energy $\Delta E(J)$ within temperature range $(0-500) \mathrm{K}$

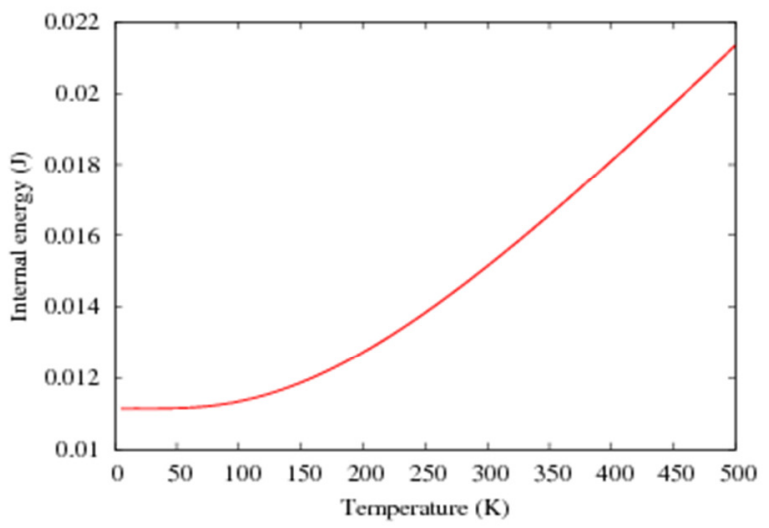

Fig. 8. The calculated Internal energy $\Delta \mathrm{F}(\mathrm{J})$ within temperature range $(0-500) \mathrm{K}$

CONCLUSION Our calculated value of $B / G$ ratio of 1.8414 shows that $\mathrm{BeS}$ is ductile and capable of being shaped or bent and drawn out. Applying the cubic stability conditions, $\mathrm{BeS}$ is found to be mechanically stable. From the computed phonon dispersion curve and the thermodynamic properties it is observed that $\mathrm{BeS}$ is both stable lattice dynamically and thermodynamically respectively.

Acknowledgements: The authors are grateful to the University of Benin for providing the workstation used in the computations and also to the Marie Curie Library of the Abdus Salam international Centre for Theoretical Physics (ICTP) for permission to use the ejds for the reference articles used in this publication. We also wish to appreciate Prof. J. O. A. Idiodi and Dr. Adetunji for providing useful resources.

\section{REFERENCES}

Baroni S., Dal Corso A., de Gironcoli S., Giannozzi P. $<$ http://www.quantumespresso.org $>$

Benosman N., Amrane N., Mecabih S., Aourag H. 2001. Structural and electronic properties of bulk BeS. Physica B 304, 214-220.

Chang J., Chen X., Zhang W., Zhu J. 2008. Firstprinciples investigations on elastic and thermodynamic properties of zinc-blende structure BeS. Chin. Phys. B 17, (4), 1377-1382.

Gonzalez-Diaz M., Rodriguez-Hernandez R., Munoz A. 1997. Elastic constants and electronic structure of beryllium chalcogenides BeS, BeSe, and BeTe from first-principles calculations. Phys. Rev. B 55. 14043-14046.

Guo L., Hu G., Zhang S., Feng W., Zhang Z. 2013. Structural, elastic, electronic and optical 
properties of beryllium chalcogenides $\mathrm{BeX}(\mathrm{X}=$ $\mathrm{S}, \mathrm{Se}, \mathrm{Te}$ ) with zinc-blende structure J. Alloys and Comp. 561, 16-22.

Heciri D., Beldi L., Drablia S., Meradji H., Derradji N. E., Belhkir H., Bouhafs B. 2007. First-principles elastic constants and electronic structure of beryllium chalcogenides $\mathrm{BeS}, \mathrm{BeSe}$ and BeTeComputational Materials Science 38, 609617.

Khenata R., Bouhemadou A., Hichour M., Baltache H., Rached D., Rerat M. 2006. Elastic and optical properties of BeS, BeSe and BeTe under pressure. Solid-State Electronics 50 1382-1388.

Kalpana G., Pari G., Mookerjee A., Bhattacharyya A. K. 1998. Ab initio Electronic Band Structure Calculations for Beryllium Chalcogenides Int. J. Mod. Phys. B 12, 1975-1984.

Luo H., Ghandehari K., Greene R. G., Ruoff A. L., Trail S. S., DiSalvo F. J.. 1995. Phase transformation of $\mathrm{BeSe}$ and $\mathrm{BeTe}$ to $\mathrm{NiAs}$ structure at high pressure. Phys. Rev. B 52, 70587064

Luo H., Ghandehari K., Greene R. G., Ruoff A. L., Trail S. S. 1994. Structural phase transformations and the equations of state of calcium chalcogenides at high pressure. Phys. Rev. B 50, 16232-16237.

Lee C., and Xavier G. 1995. Ab initio calculation of the thermodynamic properties and atomic temperature factors of $\mathrm{SiO}_{2} \propto$-quartz and stishovite. Phys. Rev. B 51 13, 8610-8613.

Laref S., and Laref A. 2012. Thermal properties of $\operatorname{BeX}(X=S$, Se and Te) compounds from ab initio quasi-harmonic method. Comput. Mater. Sci. 51, 135-140.

Mehl M. J. 1993. Pressure Dependence of the Elastic Moduli in Aluminum Rich Al-Li Compounds. Phys. Rev. B 47, 2493-2508.

Narayana C., Nesamony V. J., Ruoff A. L. 1997. Phase transformation of $\mathrm{BeS}$ and equation-of-state studies to 96 GPa. Phys. Rev. B 56, (22) 1433814343.

Okoye C. M. I. 2004. Structural, electronic, and optical properties of beryllium Monochalcogenides. Eur. Phys. J. B 39, 5-17.
Paszkowiez W., Godwod K., Domagala J., Firszi F., Szatkowski J., Meczynska H., Legowski S., Marezak M. 1998. Lattice Parameter, Microhardness And Energy Gap Of Bulk Zn 1_xBe Se Alloys Solid State Commun., 107, 12. $735-740$.

Perdew John P., Burke Kieron, Ernzerhof Matthias. 1996. Generalized Gradient Approximation Made Simple. Phys. Rev. Lett. 77, 3865-3868.

Staritzky E., Anal. Chem. 28 (1956) 915.

Srivastava G. P., Tuttincil H. M., Gunhan N.. 2004. First-principles studies of structural, electronic, and dynamical properties of Be chalcogenides. Phys. Rev. B 70. 085206-085212.

Sin'ko G. V., Smirnow N. A. 2002. Ab initio calculations of elastic constants and thermodynamic properties of bcc, fcc, and hcp Al crystals under pressure. J. Phys. Condens. Matter 14, 6989-7005.

Van Camp P. E. and Van Doren V. E. 1996. Ground state properties and structural phase transformation of beryllium sulphide. Solid State Commun. 98, 741-743.

Vassiliou M. S., Ahrens T. J. 1981. Hugoniot equation of state of periclase to 200GPa. Geophys. Res. Lett. 8, 729.

Wang S. Q. and Ye H. Q. 2003. Ab initio elastic constants for the lonsdaleite phases of $\mathrm{C}, \mathrm{Si}$ and Ge. J. Phys. Condens: Matter 15, 5307-5314.

Weir T., Vohra Y. K., Ruoff A. L. 1986. Highpressure phase transitions and the equations of state of $\mathrm{BaS}$ and $\mathrm{BaO}$. Phys. Rev. B 33, 42214226.

Yim W. M., Dismukes J. P., Stofko E. J., Paff R. J. 1972. Synthesis and some properties of BeTe, BeSe and BeS. J. Phys. Chem. Solids, 33 501505

Zachariasen W. 1926. On the crystal structure of the Telluride of beryllium, zinc, cadmium and mercury Z. Phys. Chem. 124, 277-284 ${ }^{\mathrm{a}}$.

Zachariasen W. 1926. On the crystal structure of the Telluride of beryllium, zinc, cadmium and mercury Z. Phys. Chem. 124, $440^{\mathrm{b}}$. 